\title{
On the size of linearization domains
}

\author{
BY XAVIER BUFF \\ Institut de Mathématiques, \\ Université Paul Sabatier, \\ 118, route de Narbonne, \\ 31062 Toulouse Cedex 9, France \\ AND CARSTEN L. PETERSEN \\ IMFUFA, \\ Roskilde University, \\ Univeritetsvej 1, Postbox 260 \\ DK-4000 Roskilde, Denmark
}

(Received 2 April 2007; revised 2 October 2007)

\section{Abstract}

Assume $f: U \subset \mathbb{C} \rightarrow \mathbb{C}$ is a holomorphic map fixing 0 with derivative $\lambda$, where $0<|\lambda| \leq 1$. If $\lambda$ is not a root of unity, there is a formal power series $\phi_{f}(z)=z+\mathcal{O}\left(z^{2}\right)$ such that $\phi_{f}(\lambda z)=f\left(\phi_{f}(z)\right)$. This power series is unique and we denote by $\mathrm{R}_{\text {conv }}(f) \in[0,+\infty]$ its radius of convergence. We denote by $\mathrm{R}_{\text {geom }}(f)$ the largest radius $r \in\left[0, \mathrm{R}_{\text {conv }}(f)\right]$ such that $\phi_{f}(D(0, r)) \subset U$. In this article, we present new elementary techniques for studying the maps $f \mapsto \mathrm{R}_{\text {conv }}(f)$ and $f \mapsto \mathrm{R}_{\text {geom }}(f)$. Contrary to previous approaches, our techniques do not involve studying the arithmetical properties of rotation numbers.

\section{Introduction}

Notations:

- $\mathbb{D}:=\{z \in \mathbb{C} ;|z|<1\}$,

- $\mathbb{U}:=\{z \in \mathbb{C} ;|z|=1\}$,

- $\overline{\mathbb{D}}:=\{z \in \mathbb{C} ;|z| \leq 1\}$,

- $\mathbb{D}^{*}:=\{z \in \mathbb{C} ; 0<|z| \leq 1\}$,

- $\mathbb{U}^{\circledast}:=\{z \in \mathbb{C} ;|z|=1$ and $z$ is not a root of unity $\}$,

- $\overline{\mathbb{D}}^{\circledast}:=\{z \in \mathbb{C} ; 0<|z| \leq 1$ and $z$ is not a root of unity $\}$.

In the whole article, we assume that the map $f$ is defined and holomorphic in a neighborhood of 0 with $f(0)=0$ and $f^{\prime}(0)=\lambda \in \overline{\mathbb{D}}^{\circledast}$. It is well known that when $\lambda \in \mathbb{D}^{*}$, the map $f$ is linearizable: in a neighborhood of the origin, it is analytically conjugate to its linear part

$$
L_{\lambda}: z \mapsto \lambda z
$$

If $\lambda \in \mathbb{U}^{\circledast}$, the map is formally linearizable, but the linearizing power series might fail to be convergent. 
Definition 1. If $f:(\mathbb{C}, 0) \rightarrow(\mathbb{C}, 0)$ is a holomorphic germ fixing 0 with derivative $\lambda \in \overline{\mathbb{D}}^{\circledast}$, we let $\phi_{f}(Z) \in \mathbb{C}[[Z]]$ be the unique formal power series $\phi_{f}(Z)=Z+\mathcal{O}\left(Z^{2}\right)$ such that

$$
\phi_{f} \circ L_{\lambda}=f \circ \phi_{f} .
$$

We denote by $\mathrm{R}_{\text {conv }}(f) \in[0,+\infty]$ its radius of convergence.

Our first result is the following. We shall prove it in section 2 .

Theorem 1. Suppose $\left(\lambda_{n} \in \mathbb{D}\right)$ converges to $\lambda_{0} \in \mathbb{U}^{\circledast}$ and $r>0$. If the sequence $\left(f_{n}: D(0, r) \rightarrow \mathbb{C}\right)$ with $f_{n}(0)=0$ and $f_{n}^{\prime}(0)=\lambda_{n}$ satisfies

$$
\sup _{|z|<r}\left|f_{n}(z)-\lambda_{n} z\right| \underset{n \rightarrow+\infty}{=} \mathcal{O}\left(1-\left|\lambda_{n}\right|\right)
$$

then:

- $\liminf _{n \rightarrow+\infty} \mathrm{R}_{\text {conv }}\left(f_{n}\right) \geq r$ and

- the sequence $\left(\phi_{f_{n}}\right)$ converges locally uniformly to the identity on $D(0, r)$.

Definition 2. Let $Q_{\lambda}: \mathbb{C} \rightarrow \mathbb{C}$ be the quadratic polynomial defined by

$$
Q_{\lambda}(z)=\lambda z(1+z) .
$$

Our second result is the following. We shall prove it in section 4.

Theorem 2. Assume $\left(\lambda_{n} \in \overline{\mathbb{D}}^{\circledast}\right)$ converges to $\lambda_{0} \in \mathbb{U}^{\circledast}$ with $\mathrm{R}_{\text {conv }}\left(Q_{\lambda_{n}}\right)>0$ and $\mathrm{R}_{\text {conv }}\left(Q_{\lambda_{0}}\right)>0$ and assume $r>0$. If the sequence $f_{n}: D(0, r) \rightarrow \mathbb{C}$ with $f_{n}(0)=0$ and $f_{n}^{\prime}(0)=\lambda_{n}$ converges to the rotation $L_{\lambda_{0}}$ locally uniformly on $D(0, r)$, then:

$$
\liminf _{n \rightarrow+\infty}\left(\frac{\mathrm{R}_{\text {conv }}\left(f_{n}\right)}{r} / \frac{\mathrm{R}_{\text {conv }}\left(Q_{\lambda_{n}}\right)}{\mathrm{R}_{\text {conv }}\left(Q_{\lambda_{0}}\right)}\right) \geq 1 .
$$

Remark. The condition $\mathrm{R}_{\text {conv }}\left(Q_{\lambda_{n}}\right)>0$ is automatically satisfied if $\lambda_{n} \in \mathbb{D}^{*}$.

Remark. This theorem is a form of extremality property for the quadratic family $Q_{\lambda}$. In this article, we give a very short proof of this optimality, by an enhancement of Yoccoz's methods. In the case $\lambda_{0}=\mathrm{e}^{i 2 \pi \theta_{0}}$ and $\lambda_{n}=e^{i 2 \pi \theta_{n}}$ with $\theta_{0} \in \mathbb{R}$ and $\theta_{n} \in \mathbb{R}$, this optimality follows from earlier works $[\mathbf{R i}],[\mathbf{A B C}]$ and $[\mathbf{B C 1}]$. There, as $n \rightarrow+\infty$, one bounds $\mathrm{R}_{\text {conv }}\left(f_{n}\right)$ from above and $\mathrm{R}_{\text {conv }}\left(Q_{\lambda_{n}}\right)$ from below, in terms of arithmetical properties of $\theta_{0}$ and $\theta_{n}$. Together, these results imply the aforementioned optimality. Here, we bypass the arithmetics.

Let us mention a selection of applications of our theorems.

Definition 3. If $f: U \rightarrow \mathbb{C}$ is a holomorphic map defined in a neighborhood $U$ of 0 , fixing 0 with derivative $\lambda \in \overline{\mathbb{D}}^{\circledast}$, we set

$$
\mathrm{R}_{\text {geom }}(f):=\sup \left\{r \in\left[0, \mathrm{R}_{\text {conv }}(f)\right] \mid \phi_{f}(D(0, r)) \subset U\right\}
$$

and

$$
\Delta_{f}:=\phi_{f}\left(D\left(0, \mathrm{R}_{\text {geom }}(f)\right)\right) .
$$

As we shall see in section 3 , the map $\phi_{f}$ is univalent on $D\left(0, \mathrm{R}_{\text {geom }}(f)\right)$, and so, $\mathrm{R}_{\text {geom }}(f)$ is the conformal radius of $\Delta_{f}$. 
- If $\lambda \in \mathbb{D}^{*}$, the origin is an attracting fixed point which has a basin of attraction $\Omega_{f}:=\left\{z \in U \mid f^{\circ n}(z) \rightarrow 0\right.$ as $\left.n \rightarrow+\infty\right\}$ and $\Delta_{f} \subset \Omega_{f}$.

- If $\lambda \in \mathbb{U}^{\circledast}$ and if $\mathrm{R}_{\text {conv }}(f)>0$, then $\Delta_{f}$ is the largest domain in $U$ containing 0 on which $f$ is conjugate to the rotation $L_{\lambda}$. It is the Siegel disk of $f$.

Remark. If $\Delta_{f}$ is relatively compact in $U$, then $\mathrm{R}_{\text {conv }}(f)=\mathrm{R}_{\text {geom }}(f)$. In particular, this equality holds if $f: \mathbb{C} \rightarrow \mathbb{C}$ is a polynomial fixing 0 with derivative $\lambda$. For example $\mathrm{R}_{\text {conv }}\left(Q_{\lambda}\right)=\mathrm{R}_{\text {geom }}\left(Q_{\lambda}\right)$. Also $\mathrm{R}_{\text {conv }}(f)=\mathrm{R}_{\text {geom }}(f)$ if all boundary points of $U$ are singularities of $f$.

We are interested in studying the properties of the map $f \mapsto \mathrm{R}_{\text {geom }}(f)$, and for this purpose, it will be useful to study the properties of the map $f \mapsto \mathrm{R}_{\text {conv }}(f)$. If the maps $f_{n}$ and $f_{0}$ belong to an analytic family (as in the case of the family of quadratic polynomials $Q_{\lambda}$ ), we usually only have the Lipschitz inequality

$$
\sup _{z \in K}\left|f_{n}(z)-f_{0}(z)\right| \underset{n \rightarrow+\infty}{=} \mathcal{O}\left(\left|\lambda_{n}-\lambda_{0}\right|\right)
$$

for all compact subsets $K$ of the domain of definition of $f_{0}$. In order to apply theorem 1 , we must consider non-tangential limits.

Definition 4. A sequence $\left(\lambda_{n} \in \mathbb{D}\right)$ converges to $\lambda_{0} \in \mathbb{U}$ non-tangentially if the argument of $1-\lambda_{n} / \lambda_{0}$ remains in a compact subset of $]-\pi / 2, \pi / 2[$. Equivalently,

$$
\left|\lambda_{n}-\lambda_{0}\right|=\mathcal{O}\left(1-\left|\lambda_{n}\right|\right) \text {. }
$$

Using the later formulation we shall extend this notion to sequences $\left(\lambda_{n} \in \overline{\mathbb{D}}^{\circledast}\right)$ converging to $\lambda_{0}$. Thus, such sequences are allowed to take the value $\lambda_{0}$.

We have the following corollaries of theorem 1.

Corollary 1. Suppose $\left(\lambda_{n} \in \mathbb{D}\right)$ converges non-tangentially to $\lambda_{0} \in \mathbb{U}^{\circledast}$ and $r>0$. If the sequence $\left(f_{n}: D(0, r) \rightarrow \mathbb{C}\right)$ with $f_{n}(0)=0, f_{n}^{\prime}(0)=\lambda_{n}$ satisfies

$$
\sup _{|z|<r}\left|f_{n}(z)-\lambda_{0} z\right| \underset{n \rightarrow+\infty}{=} \mathcal{O}\left(\left|\lambda_{0}-\lambda_{n}\right|\right),
$$

then:

- $\liminf _{n \rightarrow+\infty} \mathrm{R}_{\text {conv }}\left(f_{n}\right) \geq r$ and

- the sequence $\left(\phi_{f_{n}}\right)$ converges to the identity locally uniformly on $D(0, r)$.

The following result was recently obtained by Gumenuk $[\mathbf{G}]$ with different techniques.

Corollary 2. Suppose $\left(\lambda_{n} \in \mathbb{D}\right)$ converges non-tangentially to $\lambda_{0} \in \mathbb{U}^{\circledast}$ and suppose $f_{0}: U \rightarrow \mathbb{C}$ with $f_{0}(0)=0, f_{0}^{\prime}(0)=\lambda_{0}$ has a Siegel disk $\Delta_{f_{0}}$. If the sequence $\left(f_{n}: U \rightarrow \mathbb{C}\right)$ satisfies $f_{n}(0)=0, f_{n}^{\prime}(0)=\lambda_{n}$ and for every compact set $K \subset \Delta_{f_{0}}$

$$
\sup _{z \in K}\left|f_{n}(z)-f_{0}(z)\right| \underset{n \rightarrow+\infty}{=} \mathcal{O}\left(\left|\lambda_{0}-\lambda_{n}\right|\right),
$$

then:

- $\lim _{n \rightarrow+\infty} \mathrm{R}_{\text {geom }}\left(f_{n}\right)=\mathrm{R}_{\text {geom }}\left(f_{0}\right)$,

- the sequence $\left(\phi_{f_{n}}\right)$ converges to $\phi_{f_{0}}$ locally uniformly on $D\left(0, \mathrm{R}_{\text {geom }}\left(f_{0}\right)\right)$ and

- any compact set $K \subset \Delta_{f_{0}}$ is contained in $\Delta_{f_{n}}$ for $n$ large enough. 
The case $f_{n}=Q_{\lambda_{n}}$ was previously obtained by Yoccoz $[\mathbf{Y}]$. Figure 1 shows the sets $\Delta_{Q_{\lambda}}$ for different values of $\lambda \in \overline{\mathbb{D}}^{\circledast}$. As $\left(\lambda_{n} \in \mathbb{D}^{*}\right)$ converges non-tangentially to $\lambda_{0} \in \mathbb{U}$ with $\mathrm{R}_{\text {conv }}\left(Q_{\lambda_{0}}\right)>0$, the sequence of pointed domains $\left(\Delta_{Q_{\lambda_{n}}}, 0\right)$ converges in the sense of Carathéodory to the pointed domain $\left(\Delta_{Q_{\lambda_{0}}}, 0\right)$.
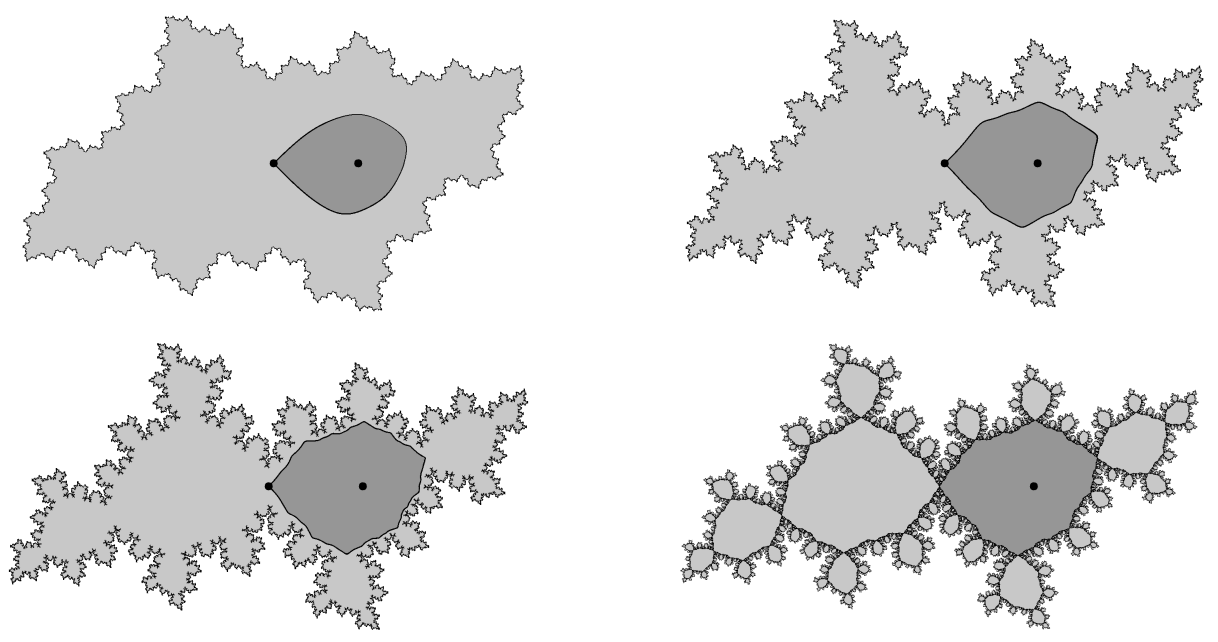

Fig. 1. The filled-in Julia sets of the polynomials $Q_{t \mathrm{e} i 2 \pi \theta}$ for $\theta:=\sqrt{2}$ and $t=.9, t=.99$, $t=.999$ and $t=1$. We have colored dark grey the sets $\Delta_{Q_{t \mathrm{e}} \mathrm{i} 2 \pi \theta}$.

Let us now come to the corollaries of theorem 2. First, we recover the following theorem of Yoccoz $[\mathbf{Y}]$. This is not surprising since our proof is largely inspired by the proof of Yoccoz.

Theorem (Yoccoz). If $\lambda_{0} \in \mathbb{U}$ satisfies $\mathrm{R}_{\text {conv }}\left(Q_{\lambda_{0}}\right)>0$ and if $f:(\mathbb{C}, 0) \rightarrow(\mathbb{C}, 0)$ is a germ fixing 0 with derivative $\lambda_{0}$, then $\mathrm{R}_{\text {conv }}(f)>0$.

Second, under the assumption $\mathrm{R}_{\text {conv }}\left(Q_{\lambda_{0}}\right)>0$, we can eliminate the conditions (1.1) and (1.2) in Corollaries 1 and 2.

Corollary 3. Suppose $\lambda_{0} \in \mathbb{U}$ satisfies $\mathrm{R}_{\text {conv }}\left(Q_{\lambda_{0}}\right)>0$, suppose $\left(\lambda_{n} \in \overline{\mathbb{D}}^{\circledast}\right)$ converges non-tangentially to $\lambda_{0}$ and suppose $r>0$. If the sequence $\left(f_{n}: D(0, r) \rightarrow \mathbb{C}\right)$ with $f_{n}(0)=0$ and $f_{n}^{\prime}(0)=\lambda_{n}$ converges to $L_{\lambda_{0}}$ uniformly on $D(0, r)$, then:

- $\liminf _{n \rightarrow+\infty} \mathrm{R}_{\text {conv }}\left(f_{n}\right) \geq r$ and

- the sequence $\left(\phi_{f_{n}}\right)$ converges to the identity locally uniformly on $D(0, r)$.

Recall that by Yoccoz's theorem, if $\lambda_{0} \in \mathbb{U}$ is such that $\mathrm{R}_{\text {conv }}\left(Q_{\lambda_{0}}\right)>0$ and if the map $f_{0}: U \rightarrow \mathbb{C}$ satisfies $f_{0}(0)=0$ and $f_{0}^{\prime}(0)=\lambda_{0}$, then $\mathrm{R}_{\text {geom }}\left(f_{0}\right)>0$ and $f_{0}$ has a Siegel disk $\Delta_{f_{0}}$.

Corollary 4. Suppose $\lambda_{0} \in \mathbb{U}$ satisfies $\mathrm{R}_{\text {conv }}\left(Q_{\lambda_{0}}\right)>0$, suppose $\left(\lambda_{n} \in \mathbb{D}\right)$ converges non-tangentially to $\lambda_{0}$ and suppose $f_{0}: U \rightarrow \mathbb{C}$ satisfies $f_{0}(0)=0$ and $f_{0}^{\prime}(0)=\lambda_{0}$. If the sequence $\left(f_{n}: U \rightarrow \mathbb{C}\right)$ with $f_{n}(0)=0$ and $f_{n}^{\prime}(0)=\lambda_{n}$ converges to $f_{0}$ locally uniformly in $U$, then:

- $\lim _{n \rightarrow+\infty} \mathrm{R}_{\text {geom }}\left(f_{n}\right)=\mathrm{R}_{\text {geom }}\left(f_{0}\right)$,

- the sequence $\left(\phi_{f_{n}}\right)$ converges to $\phi_{f_{0}}$ locally uniformly on $D\left(0, \mathrm{R}_{\text {geom }}\left(f_{0}\right)\right)$ and 
- any compact set $K \subset \Delta_{f_{0}}$ is contained in $\Delta_{f_{n}}$ for $n$ large enough.

Fourth, the following corollary was obtained by Risler [Ri] with completely different techniques. His proof relies on a careful study of the arithmetic properties of $\theta$ where $\lambda_{0}=\mathrm{e}^{i 2 \pi \theta}$. In our proof, we do not use these arithmetic properties.

Corollary 5 (Risler). Assume $\lambda_{0} \in \mathbb{U}$ satisfies $\mathrm{R}_{\text {conv }}\left(Q_{\lambda_{0}}\right)>0$ and $f_{0}: U \rightarrow \mathbb{C}$, $\left(f_{n}: U \rightarrow \mathbb{C}\right)$ satisfy $f_{0}(0)=f_{n}(0)=0, f_{0}^{\prime}(0)=f_{n}^{\prime}(0)=\lambda_{0}$. If the sequence $\left(f_{n}\right)$ converges to $f_{0}$ locally uniformly in $U$, then:

- $\lim _{n \rightarrow+\infty} \mathrm{R}_{\text {geom }}\left(f_{n}\right)=\mathrm{R}_{\text {geom }}\left(f_{0}\right)$,

- the sequence $\left(\phi_{f_{n}}\right)$ converges to $\phi_{f_{0}}$ locally uniformly on $D\left(0, \mathrm{R}_{\text {geom }}\left(f_{0}\right)\right)$ and

- any compact set $K \subset \Delta_{f_{0}}$ is contained in $\Delta_{f_{n}}$ for $n$ large enough.

Finally, we have the following consequence which can be found in $[\mathbf{C}]$.

Corollary 6. Assume $\lambda_{0} \in \mathbb{U}$ and let Poly $_{d}\left(\lambda_{0}\right)$ be the set of polynomials of degree $d$ fixing 0 with multiplier $\lambda_{0}$. If $\mathrm{R}_{\text {conv }}\left(Q_{\lambda_{0}}\right)>0$, then $\left.\mathrm{R}_{\text {conv }}: \operatorname{Poly}_{d}\left(\lambda_{0}\right) \rightarrow\right] 0,+\infty[$ is continuous.

\section{A lower bound on the size of Siegel disks}

In this section, we shall prove Theorem 1 . So, assume $\left(\lambda_{n} \in \mathbb{D}\right)$ converges to $\lambda_{0} \in \mathbb{U}^{\circledast}$ and $r>0$. Let $\left(f_{n}: D(0, r) \rightarrow \mathbb{C}\right)$ with $f_{n}(0)=0, f_{n}^{\prime}(0)=\lambda_{n}$ satisfy

$$
\sup _{|z|<r}\left|f_{n}(z)-\lambda_{n} z\right| \underset{n \rightarrow+\infty}{=} \mathcal{O}\left(1-\left|\lambda_{n}\right|\right)
$$

We will prove that for every $\rho<r$, we have $\mathrm{R}_{\text {conv }}\left(f_{n}\right) \geq \rho$ for $n$ large enough.

Lemma 1. For all $\rho<r$, there are integers $k_{0}$ and $n_{0}$ such that for all $n \geq n_{0}$,

$$
f_{n}^{\circ k_{0}}(D(0, \rho)) \subset D(0, \rho) .
$$

Proof. Set $s_{n}=1-\left|\lambda_{n}\right|$. For $n$ large enough, we can write:

$$
f_{n}(z)=\lambda_{n} z \exp \left(s_{n} \cdot u_{n}(z)\right)
$$

with $\left(u_{n}: D(0, r) \rightarrow \mathbb{C}\right)$ a sequence of holomorphic functions defined by:

$$
u_{n}(z)=\frac{1}{s_{n}} \log \left(1+\left(\frac{f_{n}(z)}{\lambda_{n} z}-1\right)\right) .
$$

The branch of logarithm is the one defined on $D(1,1)$, sending 1 to 0 , so that $u_{n}(0)=0$. In addition, our assumptions imply that the sequence $\left(u_{n}\right)$ is uniformly bounded on $D(0, r)$.

Let us fix $\rho<r$ and choose $\left.\rho^{\prime} \in\right] \rho, 1[$. For each integer $k \geq 1$, there exists an integer $n_{k}$ such that for all $n \geq n_{k}, f_{n}^{\circ k+1}$ is defined on $D\left(0, \rho^{\prime}\right)$. Then, for all $z \in D\left(0, \rho^{\prime}\right)$, we have:

$$
f_{n}^{\circ k}(z)=\lambda_{n}^{k} z \exp \left(k s_{n} \cdot u_{n}^{k}(z)\right) \quad \text { with } \quad u_{n}^{k}=\frac{1}{k} \sum_{j=0}^{k-1} u_{n} \circ f_{n}^{\circ j} .
$$

Note that $u_{n}^{k}(0)=u_{n}(0)=0$ and

$$
u_{n}^{k} \circ f_{n}=u_{n}^{k}+\frac{1}{k}\left(u_{n} \circ f_{n}^{\circ k}-u_{n}\right) .
$$


The family $\left(u_{n}^{k}: D\left(0, \rho^{\prime}\right) \rightarrow \mathbb{C}\right)_{k \geq 1, n \geq n_{k}}$ is uniformly bounded on $D\left(0, \rho^{\prime}\right)$. Let $u$ : $D\left(0, \rho^{\prime}\right) \rightarrow \mathbb{C}$ be any limit value as $k \rightarrow+\infty$. Since $f_{n} \rightarrow L_{\lambda_{0}}$, we have:

$$
u \circ L_{\lambda_{0}}=u \text {. }
$$

The function $u$ is constant on orbits under iteration of the rotation $L_{\lambda_{0}}$. Since $\lambda_{0}$ is not a root of unity, these orbits are not discrete in $D\left(0, \rho^{\prime}\right)$. Thus, $u: D\left(0, \rho^{\prime}\right) \rightarrow \mathbb{C}$ is constant. This constant is $u(0)=0$. Thus, we have proved that as $k \rightarrow+\infty$, any limit value of the family $\left(u_{n}^{k}: D\left(0, \rho^{\prime}\right) \rightarrow \mathbb{C}\right)_{k \geq 1, n \geq n_{k}}$ is constant equal to 0 .

Observe that $\frac{\log \left|\lambda_{n}^{-1}\right|}{s_{n}} \underset{n \rightarrow+\infty}{\longrightarrow} 1$. Thus, there are integers $k_{0}$ and $n_{0}$ such that

$$
\left(\forall n \geq n_{0}\right)(\forall z \in D(0, \rho)) \quad\left|u_{n}^{k_{0}}(z)\right| \leq \frac{1}{2} \frac{\log \left|\lambda_{n}^{-1}\right|}{s_{n}} .
$$

We then easily derive that

$$
\left(\forall n \geq n_{0}\right)(\forall z \in D(0, \rho)) \quad\left|f_{n}^{\circ k_{0}}(z)\right| \leq\left|\lambda_{n}\right|^{k_{0} / 2} \cdot|z|<|z| .
$$

Thus,

$$
\left(\forall n \geq n_{0}\right) \quad f_{n}^{\circ k_{0}}(D(0, \rho)) \subset D(0, \rho) .
$$

Let us fix $\rho<r$ and choose $k_{0}$ such that for all sufficiently large $n$, we have the inclusion $f_{n}^{\circ k_{0}}(D(0, \rho)) \subset D(0, \rho)$. Then, $D(0, \rho)$ belongs to the basin of attraction of 0 and the map

$$
\psi_{n}=\lim _{j \rightarrow+\infty} \frac{f_{n}^{\circ j}}{\lambda_{n}^{j}}: D(0, \rho) \rightarrow \mathbb{C}
$$

linearizes $f_{n}$. We have $\psi_{n}(0)=0$ and $\psi_{n}^{\prime}(0)=1$. In addition, $\psi_{n}$ is a limit of univalent maps. It is therefore univalent and the family $\left(\psi_{n}\right)$ is normal. Any limit value $\psi$ linearizes the rotation $L_{\lambda_{0}}$ and is therefore the identity. As a consequence, for $n$ sufficiently large, the inverse of $\psi_{n}: D(0, \rho) \rightarrow \mathbb{C}$ is defined on a Euclidean disk centered at 0 with radius arbitrarily close to $\rho$. This inverse is $\phi_{f_{n}}$. Thus, for all $\rho<r$,

$$
\liminf _{n \rightarrow+\infty} \mathrm{R}_{\text {conv }}\left(f_{n}\right) \geq \rho \text {. }
$$

The result now follows by letting $\rho$ tend to $r$.

Q.E.D.

\section{Corollaries of theorem 1}

Let us begin with some observations regarding $\mathrm{R}_{\text {geom }}(f)$.

Lemma 2. If $\lambda \in \overline{\mathbb{D}}^{\circledast}$ and $f: U \rightarrow \mathbb{C}$ is a holomorphic map fixing 0 with derivative $\lambda$, then $\phi_{f}: D\left(0, \mathrm{R}_{\text {geom }}(f)\right) \rightarrow \Delta_{f}$ is an isomorphism.

Proof. Since $\phi_{f} \circ L_{\lambda}=f \circ \phi_{f}$, we see that $f\left(\Delta_{f}\right) \subset \Delta_{f}$.

If $|\lambda|<1$, the map $\psi_{f}:=\lim _{k \rightarrow+\infty} \lambda^{-k} f^{\circ k}(z)$ is defined on $\Delta_{f}$, fixes 0 with derivative 1 and satisfies $\psi_{f} \circ f=L_{\lambda} \circ \psi_{f}$.

If $|\lambda|=1$, the sequence $\left(\psi_{m}: \Delta_{f} \rightarrow \mathbb{C}\right)_{m \geq 1}$ defined by $\psi_{m}:=\frac{1}{m} \sum_{k=0}^{m-1} \lambda^{-k} f^{\circ k}$ is 
uniformly bounded. Any limit value $\psi_{f}: \Delta_{f} \rightarrow \mathbb{C}$ fixes 0 with derivative 1 and satisfies $\psi_{f} \circ f=L_{\lambda} \circ \psi_{f}$.

In both cases, $\psi_{f} \circ \phi_{f}$ fixes 0 with derivative 1 and commutes with $L_{\lambda}$. It follows that $\psi_{f} \circ \phi_{f}$ is equal to the identity near 0 , thus on $D\left(0, \mathrm{R}_{\text {geom }}(f)\right)$ by analytic continuation. So, $\phi_{f}: D\left(0, \mathrm{R}_{\text {geom }}(f)\right) \rightarrow \Delta_{f}$ is an isomorphism with inverse $\psi_{f}$.

Lemma 3. Assume $\left(f_{n}: U \rightarrow \mathbb{C}\right)$ converges to $f_{0}: U \rightarrow \mathbb{C}$ locally uniformly, assume $f_{0}(0)=f_{n}(0)=0$ and suppose $\lambda_{0}:=f_{0}^{\prime}(0) \in \overline{\mathbb{D}}^{\circledast}$ and $\lambda_{n}:=f_{n}^{\prime}(0) \in \overline{\mathbb{D}}^{\circledast}$. Then,

$$
\limsup _{n \rightarrow+\infty} \mathrm{R}_{\text {geom }}\left(f_{n}\right) \leq \mathrm{R}_{\text {geom }}\left(f_{0}\right) .
$$

Proof. Set $\rho:=\lim \sup \mathrm{R}_{\text {geom }}\left(f_{n}\right)$ and assume, extracting a subsequence if necessary, that $\mathrm{R}_{\text {geom }}\left(f_{n}\right) \rightarrow \rho$. For any $r<\rho$, if $n$ large enough, the maps $\phi_{f_{n}}$ are defined and univalent on $D(0, r)$ and take their values in $U$. They fix 0 with derivative 1 , and thus, form a normal family. Passing to the limit on the linearizing equation $\phi_{f_{n}} \circ L_{\lambda_{n}}=f_{n} \circ \phi_{f_{n}}$, we see that any limit value $\phi: D(0, r) \rightarrow U$ linearizes $f_{0}$ :

$$
\phi \circ L_{\lambda_{0}}=f_{0} \circ \phi .
$$

Since $\phi(0)=0$ and $\phi^{\prime}(0)=1$, we see that $\phi$ is a restriction of $\phi_{f_{0}}$ and since $\phi(D(0, r)) \subset$ $U$, we see that $r \leq \mathrm{R}_{\text {geom }}\left(f_{0}\right)$. Letting $r$ tend to $\rho$, we see that

$$
\limsup _{n \rightarrow+\infty} \mathrm{R}_{\text {geom }}\left(f_{n}\right) \leq \mathrm{R}_{\text {geom }}\left(f_{0}\right) .
$$

Lemma 4. Assume $\left(f_{n}: U \rightarrow \mathbb{C}\right)$ converges to $f_{0}: U \rightarrow \mathbb{C}$ locally uniformly, assume $f_{0}(0)=f_{n}(0)=0$ and suppose $\lambda_{0}:=f_{0}^{\prime}(0) \in \mathbb{D}$ and $\lambda_{n}:=f_{n}^{\prime}(0) \in \mathbb{D}$. Then,

$$
\lim _{n \rightarrow+\infty} \mathrm{R}_{\text {geom }}\left(f_{n}\right)=\mathrm{R}_{\text {geom }}\left(f_{0}\right) .
$$

Proof. It suffices to show that $\liminf _{n \rightarrow+\infty} \mathrm{R}_{\text {geom }}\left(f_{n}\right) \geq \mathrm{R}_{\text {geom }}\left(f_{0}\right)$. Let $r<\mathrm{R}_{\text {geom }}\left(f_{0}\right)$ be arbitrary and set $\Delta^{\prime}:=\phi_{f_{0}}(D(0, r))$. If $n$ is large enough, then $f_{n}\left(\Delta^{\prime}\right)$ is relatively compact in $\Delta^{\prime}$ and the linearizing map $\psi_{f_{n}}:=\lim _{k \rightarrow+\infty} \lambda_{n}^{-k} f_{n}^{\circ k}$ is defined on $\Delta^{\prime}$. Since $f_{0}: \Delta_{f_{0}} \rightarrow \mathbb{C}$ is univalent, for $n$ large enough, the maps $f_{n}: \Delta^{\prime} \rightarrow \mathbb{C}$ are univalent. As limits of univalent maps, the maps $\psi_{f_{n}}: \Delta^{\prime} \rightarrow \mathbb{C}$ are also univalent. They fix 0 with derivative 1 and thus, belong to a normal family. Any limit value coincides with $\psi_{f_{0}}: \Delta^{\prime} \rightarrow D(0, r)$. It follows that $\liminf _{n \rightarrow+\infty} \mathrm{R}_{\text {geom }}\left(f_{n}\right) \geq r$. The result follows by letting $r$ tend to $\mathrm{R}_{\text {geom }}\left(f_{0}\right)$.

Remark. This establishes the continuity of the map $f \mapsto \mathrm{R}_{\text {geom }}(f)$ at $f_{0}$ when $f_{0}$ has an attracting fixed point at 0 .

We now come to the proof of our corollaries of theorem 1.

Corollary 1. Suppose $\left(\lambda_{n} \in \mathbb{D}\right)$ converges non-tangentially to $\lambda_{0} \in \mathbb{U}^{\circledast}$ and $r>0$. If the sequence $\left(f_{n}: D(0, r) \rightarrow \mathbb{C}\right)$ with $f_{n}(0)=0, f_{n}^{\prime}(0)=\lambda_{n}$ satisfies

$$
\sup _{|z|<r}\left|f_{n}(z)-\lambda_{0} z\right| \underset{n \rightarrow+\infty}{=} \mathcal{O}\left(\left|\lambda_{0}-\lambda_{n}\right|\right),
$$


then:

- $\liminf _{n \rightarrow+\infty} \mathrm{R}_{\mathrm{conv}}\left(f_{n}\right) \geq r$ and

- the sequence $\left(\phi_{f_{n}}\right)$ converges to the identity locally uniformly on $D(0, r)$.

Proof. If $\lambda_{n} \rightarrow \lambda_{0}$ non tangentially, then $\left|\lambda_{n}-\lambda_{0}\right|=\mathcal{O}\left(1-\left|\lambda_{n}\right|\right)$, and thus

$$
\sup _{|z|<r}\left|f_{n}(z)-\lambda_{n} z\right|=\sup _{|z|<r}\left|f_{n}(z)-\lambda_{0} z\right|+\mathcal{O}\left(\left|\lambda_{0}-\lambda_{n}\right|\right)=\mathcal{O}\left(1-\left|\lambda_{n}\right|\right) .
$$

Now, apply theorem 1.

Corollary 2. Suppose $\left(\lambda_{n} \in \mathbb{D}\right)$ converges non-tangentially to $\lambda_{0} \in \mathbb{U}^{\circledast}$ and suppose $f_{0}: U \rightarrow \mathbb{C}$ with $f_{0}(0)=0, f_{0}^{\prime}(0)=\lambda_{0}$ has a Siegel disk $\Delta_{f_{0}}$. If the sequence $\left(f_{n}: U \rightarrow \mathbb{C}\right)$ satisfies $f_{n}(0)=f_{0}(0)=0, f_{n}^{\prime}(0)=\lambda_{n}$ and for every compact set $K \subset \Delta_{f_{0}}$

$$
\sup _{z \in K}\left|f_{n}(z)-f_{0}(z)\right| \underset{n \rightarrow+\infty}{=} \mathcal{O}\left(\left|\lambda_{0}-\lambda_{n}\right|\right),
$$

then:

- $\lim _{n \rightarrow+\infty} \mathrm{R}_{\text {geom }}\left(f_{n}\right)=\mathrm{R}_{\text {geom }}\left(f_{0}\right)$,

- the sequence $\left(\phi_{f_{n}}\right)$ converges to $\phi_{f_{0}}$ locally uniformly on $D\left(0, R_{\text {geom }}\left(f_{0}\right)\right)$ and

- any compact set $K \subset \Delta_{f_{0}}$ is contained in $\Delta_{f_{n}}$ for $n$ large enough.

Proof. By lemma 3, limsup $\mathrm{R}_{\text {geom }}\left(f_{n}\right) \leq \mathrm{R}_{\text {geom }}\left(f_{0}\right)$. Thus, it is enough to prove that $\liminf _{n \rightarrow+\infty} \mathrm{R}_{\text {geom }}\left(f_{n}\right) \geq \mathrm{R}_{\text {geom }}\left(f_{0}\right)$. Let $\phi_{f_{0}}: D\left(0, \mathrm{R}_{\text {geom }}\left(f_{0}\right)\right) \rightarrow U$ be the linearizing map of $f_{0}$ fixing 0 with derivative 1 . Set

$$
g_{n}:=\phi_{f_{0}}^{-1} \circ f_{n} \circ \phi_{f_{0}} .
$$

For any $r<\mathrm{R}_{\text {geom }}\left(f_{0}\right)$, the map $g_{n}$ is eventually defined on $D(0, r)$ and the sequence of maps $\left(g_{n}: D(0, r) \rightarrow \mathbb{C}\right)$ converges uniformly to the rotation $L_{\lambda_{0}}$. More precisely,

$$
\sup _{z \in D(0, r)}\left|g_{n}(z)-\lambda_{0} z\right|=\sup _{w \in \phi_{f_{0}}(D(0, r))}\left|\psi_{f_{0}}\left(f_{n}(w)\right)-\psi_{f_{0}}\left(f_{0}(w)\right)\right|=\mathcal{O}\left(\left|\lambda_{0}-\lambda_{n}\right|\right) .
$$

By corollary $1, \liminf \mathrm{R}_{\text {conv }}\left(g_{n}\right) \geq r$ and the sequence $\left(\phi_{g_{n}}\right)$ converges uniformly on every compact subset of $D(0, r)$ to the identity. Using $\phi_{f_{n}}=\phi_{f_{0}} \circ \phi_{g_{n}}$ we see that for all $r^{\prime}<r$, if $n$ is large enough, $\phi_{f_{n}}$ is defined on $D\left(0, r^{\prime}\right)$ and takes its values in $U$ and thus, $\mathrm{R}_{\text {geom }}\left(f_{n}\right) \geq r^{\prime}$. In addition, any compact subset of $\phi_{f_{0}}(D(0, r))$ is eventually contained in $\phi_{f_{n}}(D(0, r))$. The result follows easily by letting $r$ tend to $\mathrm{R}_{\text {geom }}\left(f_{0}\right)$.

\section{Perturbations of a Brjuno rotation}

4.1. A key proposition

Recall that a quadratic-like map $g: U \rightarrow V$ is a proper map of degree 2 between two simply connected domains $U$ and $V$ with $U$ compactly contained in $V$ (see for example $[\mathbf{D H}])$.

Proposition 1. Assume $f: \mathbb{D} \rightarrow \mathbb{C}$ and $g: \mathbb{D} \rightarrow \mathbb{C}$ are holomorphic maps fixing 0 with multiplier $\lambda \in \overline{\mathbb{D}}^{\circledast}$. If $g$ is a quadratic-like map and

$$
\sup _{|z|<1}|f(z)-\lambda z|<\liminf _{|z| \rightarrow 1}|g(z)|-1 .
$$

Then, $\mathrm{R}_{\text {conv }}(f) \geq \mathrm{R}_{\text {conv }}(g)$. 
Proof. The proof to which the rest of this subsection is devoted, is a modification of a proof exposed in [BC2], which itself is a modification of a proof of Yoccoz $[\mathbf{Y}]$. The essential steps are formulated as lemmas imbedded in the proof.

Without loss of generality, we may assume that $\mathrm{R}_{\text {conv }}(g)>0$ since otherwise, the result is trivial. Consider the one-parameter families of maps

$$
\left\{f_{a}: D\left(0, \min \left(1,|a|^{-1}\right)\right) \rightarrow \mathbb{C}\right\}_{a \in \mathbb{C}} \quad \text { and } \quad\left\{g_{b}: D\left(0, \min \left(1,|b|^{-1}\right)\right) \rightarrow \mathbb{C}\right\}_{b \in \mathbb{C}}
$$

defined by:

$$
f_{a}(z)=f(z)-\lambda z+\frac{1}{a} g(a z) \quad \text { and } \quad g_{b}(w)=\frac{1}{b} f_{1 / b}(b w)=\frac{1}{b} f(b w)-\lambda w+g(w) .
$$

The family $f_{a}$ extends analytically at $a=0$ by $f_{0}=f$ and the family $g_{b}$ extends analytically at $b=0$ by $g_{0}=g$. We have:

$$
\left(\forall b \in \mathbb{C}^{*}\right) \quad \mathrm{R}_{\text {conv }}\left(g_{b}\right)=\frac{1}{|b|} \mathrm{R}_{\text {conv }}\left(f_{1 / b}\right) .
$$

Set

$$
U_{b}:=\left\{z \in \mathbb{D} \mid g_{b}(z) \in \mathbb{D}\right\} .
$$

Lemma 5. When $|b| \leq 1$, the restriction $g_{b}: U_{b} \rightarrow \mathbb{D}$ is quadratic-like.

Proof. Since $g_{0}: \mathbb{D} \rightarrow \mathbb{C}$ is quadratic-like, $U_{0}=g_{0}^{-1}(\mathbb{D})$ is compactly contained in $\mathbb{D}$ and every point in $\mathbb{D}$ has exactly two preimages by $g_{0}$ in $\mathbb{D}$, counting multiplicities. Schwarz's lemma and Rouché's theorem implies that for $|b| \leq 1, U_{b}$ is compactly contained in $\mathbb{D}$ and every point in $\mathbb{D}$ has exactly two preimages by $g_{b}$ in $\mathbb{D}$, counting multiplicities. It follows that $g_{b}: U_{b} \rightarrow \mathbb{D}$ is a proper map of degree 2 . Note that $U_{b}$ is connected since otherwise, the component of $U_{b}$ containing 0 would be mapped biholomorphically to $\mathbb{D}$, which, by Schwarz's lemma, is not possible since $|\lambda| \leq 1$.

This lemma has the following consequence.

Lemma 6. The map $b \mapsto \log \mathrm{R}_{\text {conv }}\left(g_{b}\right)$ is harmonic in a neighborhood of $\overline{\mathbb{D}}$.

Proof. If $|\lambda|<1$, we may argue as follows. We can find $r_{0}>1$ so that when $|b|<r_{0}$, the map $g_{b}: U_{b} \rightarrow \mathbb{D}$ is a quadratic-like map. It has an attracting fixed point at 0 with multiplier $\lambda$. Let $\omega_{b}$ be the unique critical point of $g_{b}$ in $U_{b}$, let $\Omega_{b}$ be the basin of attraction of 0 and let $\psi_{b}: \Omega_{b} \rightarrow \mathbb{C}$ be the linearizing map defined by: $\psi_{b}=\lim _{n \rightarrow+\infty} \lambda^{-n} g_{b}^{\text {on }}$. Then, $\phi_{g_{b}}: D\left(0, \mathrm{R}_{\text {conv }}\left(g_{b}\right)\right) \rightarrow \mathbb{D}$ is univalent with inverse $\psi_{b}$ and $\mathrm{R}_{\text {conv }}\left(g_{b}\right)=\left|\psi_{b}\left(\omega_{b}\right)\right|$. The map $b \mapsto \psi_{b}\left(\omega_{b}\right)$ is holomorphic in $D\left(0, r_{0}\right)$ and so, the map $b \mapsto \log \mathrm{R}_{\text {conv }}\left(g_{b}\right)$ is harmonic in $D\left(0, r_{0}\right)$.

If $|\lambda|=1$, we may argue as follows. We can find $r_{0}>1$ and $t_{0}<1$ so that when $t_{0}<t \leq 1$ and $|b|<r_{0}$, the map $g_{b, t}: U_{b} \rightarrow D(0, t)$ defined by

$$
g_{b, t}(w)=t \cdot g_{b}(w)
$$

is a quadratic-like map. This quadratic-like map has an attracting fixed point at 0 with multiplier $t \lambda$ converging non tangentially to $\lambda$ as $t \rightarrow 1$. As previously,

$$
\mathrm{R}_{\text {conv }}\left(g_{b, t}\right)=\left|\psi_{b, t}\left(\omega_{b, t}\right)\right| .
$$

with $\omega_{b, t}$ the unique critical point of $g_{b, t}$ in $U_{b}$ and $\psi_{b, t}: \Omega_{b, t} \rightarrow \mathbb{C}$ the linearizing map defined by $\psi_{b, t}=\lim _{n \rightarrow+\infty}(t \lambda)^{-n} g_{b, t}^{\circ n}$. 
Consider the family of holomorphic maps

$$
\left\{u_{t}: D\left(0, r_{0}\right) \rightarrow \mathbb{D}^{*}\right\}_{t_{0}<t<1}
$$

defined by:

$$
u_{t}(b)=\psi_{b, t}\left(\omega_{b, t}\right) .
$$

This family is normal and there is a sequence $\left.t_{n} \in\right] t_{0}, 1[$ converging to 1 such that the sequence $\left(u_{t_{n}}\right)$ converges uniformly on every compact subset of $D\left(0, r_{0}\right)$ to a limit $u_{1}: D\left(0, r_{0}\right) \rightarrow \overline{\mathbb{D}}$ which either identically vanishes, or does not vanish.

By Corollary 2 and Lemma 4 , we have ${ }^{1}$

$$
\forall b \in D\left(0, r_{0}\right) \quad \log \mathrm{R}_{\text {conv }}\left(g_{b}\right)=\log \left|u_{1}(b)\right| .
$$

Since we assumed that $\mathrm{R}_{\text {conv }}\left(g_{0}\right)=\mathrm{R}_{\text {geom }}(g)>0, u_{1}$ does not vanish on $D\left(0, r_{0}\right)$ and $b \mapsto \log \mathrm{R}_{\text {conv }}\left(g_{b}\right)$ is harmonic in $D\left(0, r_{0}\right)$.

Let avg $m(a)$ denote the average of the function $m(a)$ on the circle $|a|=r$ (with $|a|=r$

respect to the Lebesgue measure on the circle). As an immediate consequence of lemma 6 , we have the following equality:

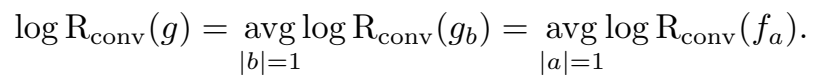

The following result is extracted from $[\mathbf{B C 2}]$. We include it here for completeness.

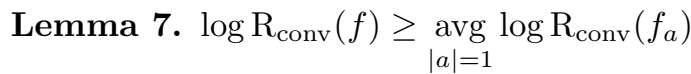

Proof. Look at the formal linearizing power series of $f_{a}$ :

$$
\phi_{f_{a}}(Z)=Z+\sum_{n=2}^{+\infty} b_{n}(a) Z^{n} .
$$

By Hadamard's theorem,

$$
\frac{1}{\mathrm{R}_{\text {conv }}\left(f_{a}\right)}=\limsup _{n \rightarrow+\infty} \sqrt[n]{\left|b_{n}(a)\right|}
$$

The coefficients $b_{n}(a)$ are polynomials in $a$. Thus

$$
\frac{1}{n} \log \left|b_{n}(0)\right| \leq \underset{|a|=1}{\operatorname{avg}} \frac{1}{n} \log \left|b_{n}(a)\right|
$$

By lemma 5 , for $|a|=1$, the map $f_{a}$ has a quadratic-like restriction $f_{a}: \frac{1}{a} U_{1 / a} \rightarrow \mathbb{D}$. In that case, the linearizing map $\phi_{f_{a}}$ takes its values in $\mathbb{D}$ and it follows from the Cauchy inequalities that

$$
\left|b_{n}(a)\right| \leq \frac{1}{\left(\mathrm{R}_{\text {conv }}\left(f_{a}\right)\right)^{n}} .
$$

Recall that $b=1 / a$. We have seen that $b \mapsto \mathrm{R}_{\text {conv }}\left(g_{b}\right)$ is continuous and non-vanishing on $\overline{\mathbb{D}}$. Thus, when $|a|=1, \mathrm{R}_{\text {conv }}\left(f_{a}\right)=\mathrm{R}_{\text {conv }}\left(g_{b}\right)$ reaches a minimum $c>0$ and

$$
\frac{1}{n} \log \left|b_{n}(a)\right| \leq \log \frac{1}{\mathrm{R}_{\mathrm{conv}}\left(f_{a}\right)} \leq \log \frac{1}{c} .
$$

1 Note that since the maps $g_{b}: U_{b} \rightarrow \mathbb{D}$ and $g_{b, t}: U_{b} \rightarrow D(0, t)$ are quadratic-like, we have $\mathrm{R}_{\text {conv }}\left(g_{b}\right)=\mathrm{R}_{\text {geom }}\left(g_{b}\right)$ and $\mathrm{R}_{\text {conv }}\left(g_{b, t}\right)=\mathrm{R}_{\text {geom }}\left(g_{b, t}\right)$. 
This uniform upper bound allows us to apply Fatou's lemma:

$$
\begin{aligned}
& -\log \mathrm{R}_{\text {conv }}(f)=\limsup _{n \rightarrow+\infty} \frac{1}{n} \log \left|b_{n}(0)\right| \leq \limsup _{n \rightarrow+\infty} \operatorname{avg} \frac{1}{n} \log \left|b_{n}(a)\right| \\
& \leq \underset{|a|=1}{\operatorname{avg} \limsup } \frac{1}{n \rightarrow+\infty} \log \left|b_{n}(a)\right|=-\underset{|a|=1}{\operatorname{avg}} \log \mathrm{R}_{\mathrm{conv}}\left(f_{a}\right) .
\end{aligned}
$$

Equality $4 \cdot 2$ and lemma 7 yield:

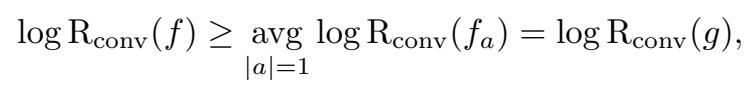

whence $\mathrm{R}_{\text {conv }}(f) \geq \mathrm{R}_{\text {conv }}(g)$. This completes the proof of Proposition 1 .

\subsection{Proof of theorem 2}

Our standing assumption is that $\lambda_{0} \in \mathbb{U}$ is such that $\mathrm{R}_{\text {conv }}\left(Q_{\lambda_{0}}\right)>0$. In that case, we say that the rotation $L_{\lambda_{0}}$ is a Brjuno rotation. ${ }^{2}$

We assume $\left(f_{n}: D(0, r) \rightarrow \mathbb{C}\right)_{n \geq 0}$ is a sequence of maps such that

- $f_{n}(0)=0$,

- $f_{n}^{\prime}(0)=\lambda_{n} \in \overline{\mathbb{D}}^{\circledast}$ and

- the sequence $\left(f_{n}\right)$ converges to the rotation $L_{\lambda_{0}}$ uniformly on every compact subset of $D(0, r)$.

We want to show that

$$
\liminf _{n \rightarrow+\infty}\left(\frac{\mathrm{R}_{\text {conv }}\left(f_{n}\right)}{r} / \frac{\mathrm{R}_{\text {conv }}\left(Q_{\lambda_{n}}\right)}{\mathrm{R}_{\text {conv }}\left(Q_{\lambda_{0}}\right)}\right) \geq 1 .
$$

Let $K_{\lambda}$ be the filled-in Julia set of $Q_{\lambda}$ and böt ${ }_{\lambda}: \mathbb{C} \backslash K_{\lambda} \rightarrow \mathbb{C} \backslash \overline{\mathbb{D}}$ be the Böttcher coordinate conjugating $Q_{\lambda}$ to $z \mapsto z^{2}$. Let $G_{\lambda}: \mathbb{C} \rightarrow[0,+\infty[$ be the Green function of $Q_{\lambda}$ :

$$
G_{\lambda}(z):= \begin{cases}0 & \text { if } z \in K_{\lambda} \\ \log \left|\operatorname{böt}_{\lambda}(z)\right| & \text { if } z \in \mathbb{C} \backslash K_{\lambda} .\end{cases}
$$

For $\eta>0$, set

$$
U_{\lambda, \eta}:=\left\{z \in \mathbb{C} \mid G_{\lambda}(z)<\eta\right\}
$$

By Schwarz's reflection principle, any conformal representation $\chi_{\lambda, \eta}: U_{\lambda, \eta} \rightarrow \mathbb{D}$ sending 0 to 0 extends univalently to $U_{\lambda, 2 \eta}$. Indeed, if $\mathrm{e}^{\eta} \leq|z|<\mathrm{e}^{2 \eta}$, then $1<\left|\mathrm{e}^{2 \eta} / \bar{z}\right| \leq \mathrm{e}^{\eta}$ and we can set

$$
\chi_{\lambda, \eta}\left(\mathrm{böt}_{\lambda}^{-1}(z)\right)=s \circ \chi_{\lambda, \eta} \circ \mathrm{böt}_{\lambda}^{-1}\left(\frac{\mathrm{e}^{2 \eta}}{\bar{z}}\right) \quad \text { with } \quad s(z)=\frac{1}{\bar{z}} .
$$

Denote by $V_{\lambda, \eta}$ the image of the extended map $\chi_{\lambda, \eta}: U_{\lambda, 2 \eta} \rightarrow \mathbb{C}$. The isomorphism $\chi_{\lambda, \eta}: U_{\lambda, 2 \eta} \rightarrow V_{\lambda, \eta}$ conjugates $Q_{\lambda}: U_{\lambda, \eta} \rightarrow U_{\lambda, 2 \eta}$ to a quadratic-like map $g_{\lambda, \eta}: \mathbb{D} \rightarrow V_{\lambda, \eta}$.

${ }^{2}$ Yoccoz $[\mathbf{Y}]$ proved that $Q_{\mathrm{e}^{i 2 \pi \theta}}$ is linearizable if and only if $\theta$ satisfies an arithmetic condition known as the Brjuno condition. 
The modulus of the annulus $V_{\lambda, \eta} \backslash \overline{\mathbb{D}}$ is equal to $\eta /(2 \pi)$ and thus, there is a constant $c(\eta)>0$ such that

$$
(\forall \lambda \in \overline{\mathbb{D}}) \quad \liminf _{|z| \rightarrow 1}\left|g_{\lambda, \eta}(z)\right|>1+c(\eta) .
$$

If $U \subset \mathbb{C}$ is a simply connected domain containing 0 , we denote by $\operatorname{Rad}(U)$ its conformal radius at 0 , i.e. $\operatorname{Rad}(U)=\left|1 / \chi^{\prime}(0)\right|$ for any isomorphism $\chi: U \rightarrow \mathbb{D}$ fixing 0 .

Now, given $\rho<r$, if $n$ is large enough, the map $F_{n}: z \mapsto \rho^{-1} f_{n}(\rho z)$ is defined and holomorphic on a neighborhood of $\overline{\mathbb{D}}$ and

$$
\sup _{|z|=1}\left|F_{n}(z)-\lambda_{n} z\right|<c(\eta) \text {. }
$$

By proposition 1, we see that for all $\eta>0$ and all $\rho<r$, if $n$ is large enough,

$$
\frac{\mathrm{R}_{\text {conv }}\left(f_{n}\right)}{\rho}=\mathrm{R}_{\text {conv }}\left(F_{n}\right) \geq \mathrm{R}_{\text {conv }}\left(g_{\lambda_{n}, \eta}\right)=\frac{\mathrm{R}_{\text {conv }}\left(Q_{\lambda_{n}}\right)}{\operatorname{Rad}\left(U_{\lambda_{n}, \eta}\right)} .
$$

As $n$ tends to infinity, $\operatorname{Rad}\left(U_{\lambda_{n}, \eta}\right)$ tends to $\operatorname{Rad}\left(U_{\lambda_{0}, \eta}\right)$. So, this can be rewritten as

$$
(\forall \eta>0)(\forall \rho<r) \quad \liminf _{n \rightarrow+\infty} \mathrm{R}_{\text {conv }}\left(f_{n}\right) \cdot \frac{\operatorname{Rad}\left(U_{\lambda_{0}, \eta}\right)}{\mathrm{R}_{\text {conv }}\left(Q_{\lambda_{n}}\right)} \geq \rho .
$$

As $\eta$ tends to $0, \operatorname{Rad}\left(U_{\lambda_{0}, \eta}\right)$ tends to $\mathrm{R}_{\text {conv }}\left(Q_{\lambda_{0}}\right)$. So, letting $\eta$ tend to 0 and $\rho$ tend to $r$, we obtain the required result:

$$
\liminf _{n \rightarrow+\infty} \mathrm{R}_{\text {conv }}\left(f_{n}\right) \cdot \frac{\mathrm{R}_{\text {conv }}\left(Q_{\lambda_{0}}\right)}{\mathrm{R}_{\text {conv }}\left(Q_{\lambda_{n}}\right)} \geq r
$$

This completes the proof of theorem 2 .

\section{Corollaries of theorem 2}

Let us begin with Yoccoz's theorem.

Theorem (Yoccoz). If $\lambda_{0} \in \mathbb{U}$ is such that $\mathrm{R}_{\text {conv }}\left(Q_{\lambda_{0}}\right)>0$ and if $f:(\mathbb{C}, 0) \rightarrow(\mathbb{C}, 0)$ is a germ fixing 0 with derivative $\lambda_{0}$, then $\mathrm{R}_{\text {conv }}(f)>0$.

Proof. Choose $\lambda_{n}=\lambda_{0}$ and define $f_{n}:(\mathbb{C}, 0) \rightarrow(\mathbb{C}, 0)$ by

$$
f_{n}(z)=\frac{1}{r_{n}} f\left(r_{n} z\right)
$$

where $\left(r_{n}\right)$ is a sequence converging to 0 . Then, $\left(f_{n}\right)$ converges locally uniformly in $\mathbb{C}$ to the rotation $L_{\lambda_{0}}$. Therefore,

$$
\liminf _{n \rightarrow+\infty} \frac{\mathrm{R}_{\text {conv }}(f)}{r_{n}}=\liminf _{n \rightarrow+\infty} \mathrm{R}_{\text {conv }}\left(f_{n}\right)=+\infty .
$$

Thus, $\mathrm{R}_{\text {conv }}(f)>0$.

Let us now come to the proofs of our corollaries of theorem 2 .

Corollary 3. Suppose $\lambda_{0} \in \mathbb{U}$ satisfies $\mathrm{R}_{\text {conv }}\left(Q_{\lambda_{0}}\right)>0$, suppose $\left(\lambda_{n} \in \overline{\mathbb{D}}^{\circledast}\right)$ converges non-tangentially to $\lambda_{0}$ and supose $r>0$. If the sequence $\left(f_{n}: D(0, r) \rightarrow \mathbb{C}\right)$ with $f_{n}(0)=0$ and $f_{n}^{\prime}(0)=\lambda_{n}$ converges to $L_{\lambda_{0}}$ uniformly on $D(0, r)$, then:

- $\liminf _{n \rightarrow+\infty} \mathrm{R}_{\text {conv }}\left(f_{n}\right) \geq r$ and 
- the sequence $\left(\phi_{f_{n}}\right)$ converges to the identity locally uniformly on $D(0, r)$.

Proof. Assume $\left(\lambda_{n} \in \overline{\mathbb{D}}^{\circledast}\right)$ converges non tangentially to $\lambda_{0}$. According to Corollary 2, $\mathrm{R}_{\text {conv }}\left(Q_{\lambda_{n}}\right)=\mathrm{R}_{\text {geom }}\left(Q_{\lambda_{n}}\right)$ converges to $\mathrm{R}_{\text {conv }}\left(Q_{\lambda_{0}}\right)=\mathrm{R}_{\text {geom }}\left(Q_{\lambda_{0}}\right)$. Thus, by Theorem 2 , $\liminf _{n \rightarrow+\infty} R_{\text {conv }}\left(f_{n}\right) \geq r$.

The space of univalent maps on $D(0, r)$, fixing the origin with derivative 1 , is compact. The map $\phi_{f_{n}}$ is univalent on $D\left(0, \mathrm{R}_{\text {geom }}\left(f_{n}\right)\right)$ and fixes the origin with derivative 1 . We will show that $\liminf _{n \rightarrow+\infty} \mathrm{R}_{\text {geom }}\left(f_{n}\right) \geq r$. Then, any limit map of the sequence $\left(\phi_{f_{n}}\right)$ is the identity on $D(0, r)$, because it conjugates $L_{\lambda_{0}}$ to itself. Thus, the whole sequence $\left(\phi_{f_{n}}\right)$ converges to the identity locally uniformly on $D(0, r)$.

To see $\liminf _{n \rightarrow+\infty} \mathrm{R}_{\text {geom }}\left(f_{n}\right) \geq r$, choose a function $g$ holomorphic in $D(0, r)$ such that all points of modulus $r$ are singularities of $g$. By upper semicontinuity of $\mathrm{R}_{\text {geom }}$ (Lemma 3 ), if $\varepsilon$ is small enough, then $\mathrm{R}_{\text {geom }}\left(f_{n}+\varepsilon z^{2} g\right) \leq \mathrm{R}_{\text {geom }}\left(f_{n}\right)+1 / n$ and for all but countably many values of $\varepsilon$ all points of modulus $r$ are singularities of $f_{n}+\varepsilon z^{2} g$. Thus we can choose a sequence $\left(\varepsilon_{n}\right)$ converging to 0 such that $g_{n}=f_{n}+\varepsilon_{n} z^{2} g$ satisfies:

(i) $g_{n} \rightarrow L_{\lambda}$ locally uniformly in $D(0, r)$.

(ii) $\mathrm{R}_{\text {geom }}\left(g_{n}\right)=\mathrm{R}_{\text {conv }}\left(g_{n}\right)$ by the Remark following Definition 3 .

(iii) $\mathrm{R}_{\text {geom }}\left(g_{n}\right) \leq \mathrm{R}_{\text {geom }}\left(f_{n}\right)+1 / n$.

Hence

$$
\liminf _{n \rightarrow+\infty} \mathrm{R}_{\text {geom }}\left(f_{n}\right) \geq \liminf _{n \rightarrow+\infty} \mathrm{R}_{\text {geom }}\left(g_{n}\right)=r
$$

where the inequality follows from (iii) and the equality follows by combining Theorem 2 and (ii).

Corollary 4. Suppose $\lambda_{0} \in \mathbb{U}$ satisfies $\mathrm{R}_{\text {conv }}\left(Q_{\lambda_{0}}\right)>0$, suppose $\left(\lambda_{n} \in \mathbb{D}\right)$ converges non-tangentially to $\lambda_{0}$ and suppose $f_{0}: U \rightarrow \mathbb{C}$ satisfies $f_{0}(0)=0$ and $f_{0}^{\prime}(0)=\lambda_{0}$. If the sequence $\left(f_{n}: U \rightarrow \mathbb{C}\right)$ with $f_{n}(0)=0$ and $f_{n}^{\prime}(0)=\lambda_{n}$ converges to $f_{0}$ locally uniformly in $U$, then:

- $\lim _{n \rightarrow+\infty} \mathrm{R}_{\text {geom }}\left(f_{n}\right)=\mathrm{R}_{\text {geom }}\left(f_{0}\right)$,

- the sequence $\left(\phi_{f_{n}}\right)$ converges to $\phi_{f_{0}}$ locally uniformly on $D\left(0, R_{\text {geom }}\left(f_{0}\right)\right)$ and

- any compact set $K \subset \Delta_{f_{0}}$ is contained in $\Delta_{f_{n}}$ for $n$ large enough.

Proof. Similar to the proof of Corollary 2, Corollary 1 being replaced by Corollary 3.

Corollary 5 (Risler). Assume $\lambda_{0} \in \mathbb{U}$ satisfies $\mathrm{R}_{\text {conv }}\left(Q_{\lambda_{0}}\right)>0$ and $f_{0}: U \rightarrow \mathbb{C}$, $\left(f_{n}: U \rightarrow \mathbb{C}\right)$ satisfy $f_{0}(0)=f_{n}(0)=0, f_{0}^{\prime}(0)=f_{n}^{\prime}(0)=\lambda_{0}$. If the sequence $\left(f_{n}\right)$ converges to $f_{0}$ locally uniformly in $U$, then:

- $\lim _{n \rightarrow+\infty} \mathrm{R}_{\text {geom }}\left(f_{n}\right)=\mathrm{R}_{\text {geom }}\left(f_{0}\right)$,

- the sequence $\left(\phi_{f_{n}}\right)$ converges to $\phi_{f_{0}}$ locally uniformly on $D\left(0, \mathrm{R}_{\text {geom }}\left(f_{0}\right)\right)$ and

- any compact set $K \subset \Delta_{f_{0}}$ is contained in $\Delta_{f_{n}}$ for $n$ large enough.

Proof. Similar to the proof of Corollary 4.

Corollary 6. Assume $\lambda_{0} \in \mathbb{U}$ and let $\operatorname{Poly}_{d}\left(\lambda_{0}\right)$ be the set of polynomials of degree $d$ fixing 0 with multiplier $\lambda_{0}$. If $\mathrm{R}_{\text {conv }}\left(Q_{\lambda_{0}}\right)>0$, then $\left.\mathrm{R}_{\text {conv }}: \operatorname{Poly}_{d}\left(\lambda_{0}\right) \rightarrow\right] 0,+\infty[$ is continuous. 
Proof. By Yoccoz's theorem, $\mathrm{R}_{\text {conv }}(P)>0$ for all $P \in \operatorname{Poly}_{d}\left(\lambda_{0}\right)$. The linearizing maps $\phi_{P}: D\left(0, \mathrm{R}_{\text {conv }}(P)\right) \rightarrow \mathbb{C}$ are univalent and take their values in the filled-in Julia set of $P$ : they are conformal isomorphisms between the disk $D\left(0, \mathrm{R}_{\text {conv }}(P)\right)$ and the Siegel disk $\Delta_{P}$. It follows that $\mathrm{R}_{\text {conv }}(P)<+\infty$ for all $P \in \operatorname{Poly}_{d}\left(\lambda_{0}\right)$. The result now follows from corollary 5 since $\mathrm{R}_{\text {conv }}(P)=\mathrm{R}_{\text {geom }}(P)$.

Acknowledgements. We would like to thank Arnaud Chéritat for helpful discussions.

\section{REFERENCES}

[ABC] A. Avila, X. Buff and A. Chéritat. Siegel disks with smooth boundaries. Acta Math. 193 (2004) 1-30.

[BC1] X. Buff and A. ChÉritat. The Brjuno Function Continuously Estimates the Size of Siegel Disks. Annals of Math. 164 (2006) 265-312.

[BC2] X. Buff and A. Chéritat. A new proof of a conjecture of Yoccoz. Preprint (2007).

[C] A. ChÉRItat. Recherche d'ensembles de Julia de mesure de Lebesgue positive. PhD. Thesis (2001).

[DH] A. Douady and J. H. Hubbard. On the dynamics of polynomial-like mappings. Ann. Sci. ENS Paris 18 (1985) 287-343.

[G] P. Gumenuk Carathéodory convergence of immediate basins of attraction to a Siegel disk. Preprint (2006).

[Ri] E. RISLER. Linéarisation des perturbations holomorphes des rotations et applications. Mém. Soc. Math. Fr. (N.S.) $\mathbf{7 7}$ (1999).

[Y] J.C. Yoccoz. Petits diviseurs en dimension 1. Astérisque 231 (1995). 